ISSN electrónico: 2445-1355

DOI: https://dx.doi.org/10.14201/fj2020527382

\title{
APLICACIÓN DEL MÉTODO STRONG-LAMP PARA LA DETECCIÓN MOLECULAR DE STRONGYLOIDES STERCORALIS EN UNA POBLACIÓN DE PORTO IGUAZÚ, MISIONES, ARGENTINA
}

\author{
Application of the Strong-LAMP Method for \\ the Molecular Detection of Strongyloides Stercoralis in \\ a Population of Porto Iguazú, Misiones, Argentina
}

Carmen RODRÍGUEZ DIEGO; Pedro FERNÁNDEZ-SOTO; Antonio MURO ÁLVAREZ

Departamento de Biología Animal, Parasitología, Ecología, Edafología y Química agrícola. Unidad de Investigación Enfermedades Infecciosas y Tropicales (e-INTRO). Instituto de Investigación Biomédica de Salamanca-Centro de Investigación de Enfermedades Tropicales de la Universidad de Salamanca (IBSAL-CIETUS). Facultad de Farmacia. Universidad de Salamanca. Campus Miguel de Unamuno, s/n. Avda. Ldo. Méndez Nieto, s/n. 37007 Salamanca. España

Correo-e: cietus@usal.es

RESUMEN: Strongiloides stercoralis es un parásito nematodo de distribución global y el principal agente causal de la estrongiloidiasis. La infección crónica presenta un curso asintomático, sin embargo, puede producir síndrome de hiperinfección en pacientes inmunodeprimidos. En la actualidad, la falta de un diagnóstico estandarizado genera una subestimación de la prevalencia de la enfermedad. El análisis coprológico, considerado como el gold-standard, presenta una muy baja sensibilidad. Una alternativa es el método LAMP específico y sensible para la detección de S. stercoralis, el Strong-LAMP. En el presente estudio, se ha utilizado un total de 100 muestras de heces recolectadas de una población de Porto Iguazú, Misiones (Argentina) y almacenadas en papel de filtro hasta su analisis. Cuando se aplicó el Strong-LAMP para la detección de ADN de Strongyloides spp., hasta 39 muestras $(39 \%)$ resultaron positivas, incluyendo 5 positivas al

Ediciones Universidad de Salamanca / @@ఠ $\quad$ FarmaJournal, vol. 5, núm. 2 (2020), pp. 73-82 
análisis coprológico, frente a 7 muestras $(7 \%)$ positivas obtenidas por análisis parasitológico. Por tanto, el Strong-LAMP ha demostrado ser un método más sensible para la detección de Strongyloides stercoralis en muestras de heces almacenadas en papel de filtro que los métodos de diagnóstico parasitológicos y podría utilizarse en combinación con los métodos parasitológicos en áreas endémicas para establecer con mayor fiabilidad la prevalencia real de la enfermedad.

Palabras clave: Estrongiloidiasis; Strong-LAMP; diagnóstico molecular; muestras de heces; papel de filtro.

ABSTRACT: Strongiloides stercoralis is a nematode globally distributed and the chief causative agent of human Strongyloidiasis. Chronic infection is clinically asymptomatic, but, it can cause Hyperinfection Syndrome in immunocompromised patients. At present, the lack of a standardized diagnosis generates an underestimation of the prevalence of the disease. Coprological test, considered as the gold-standard, has a very low sensitivity. An alternative is the specific and sensitive LAMP method for the detection of S. stercoralis, the Strong-LAMP. In the present study, it have been used a total of 100 stool samples collected from a population of Porto Iguazú, Misiones (Argentina) and stored on filter paper until analysis. When StrongLAMP was applied for the detection of Strongyloides spp. DNA, up to 39 samples $(39 \%)$ were positive, including 5 positive obtained by coprological test, compared to 7 positive samples $(7 \%)$ obtained by parasitological test. Therefore, Strong-LAMP has demonstrated more sensitive method for the detection of Strongyloides stercoralis in stool samples stored in filter paper than parasitological diagnostic methods and could be used in combination with parasitological methods in endemic areas to establish with greater reliability the real prevalence of the disease.

Keywords: Strongyloidiasis; Strong-LAMP; molecular diagnosis; stool samples; filter paper.

\section{INTRODUCCIÓN}

La estrongiloidiasis es una parasitosis humana causada por el nematodo Strongyloides stercoralis; se han descrito infecciones también por S. fuelleborni fuelleborni en África y S. fuelleborni kellyi en Papúa Nueva Guinea (King et al., 2004). S. stercolaris es un parásito que se transmite por el suelo (geohelminto), con un ciclo de vida particular y muy complejo en el que se presentan dos formas de 
transmisión: heteroinfección (transmisión mediante la penetración transcutánea de larvas filariformes) y autoinfección (transformación en el intestino grueso de larvas rabditiformes en larvas filariformes). La autoinfección es una característica biológica exclusiva del género Strongyloides spp. (Faust et al., 1983).

Se estima una prevalencia global de aproximadamente 30-100 millones de personas infectadas con $S$. stercoralis a nivel mundial, presentando una distribución cosmopolita, fundamentalmente en áreas tropicales y subtropicales de América del Norte y del Sur, Asia y África subsahariana (Schär et al., 2013). Actualmente la estrongiloidiasis está considerada por la OMS como una de las 20 enfermedades olvidadas o desatendidas (Neglected Tropical Diseases; NTDs) (https://www. who.int/intestinal_worms/epidemiology/strongyloidiasis/en/).

Las manifestaciones clínicas de la estrongiloidiasis dependen de la etapa de invasión y desarrollo del parásito, de su capacidad de autoinfección y del estado inmunológico del paciente, pudiendo aparecer como una infección aguda, una infección crónica, producir el síndrome de hiperinfección y/o una infección diseminada. La estrongiloidiasis aguda es poco común y suele presentarse en viajeros que regresan de una zona endémica, con dermatitis pruriginosa (por la penetración de larvas por la piel), neumonitis con tos y expectoración (al pasar las larvas por los pulmones) y fiebre. Cuando los parásitos alcanzan el intestino se produce dolor gastrointestinal acompañado de diarrea, náuseas y, ocasionalmente, vómitos. La estrongiloidiasis crónica suele ser asintomática, aunque también puede presentar una sintomatología leve o moderada, con manifestaciones gastrointestinales, pulmonares, cutáneas y eosinofilia (presente en el $75 \%$ de los pacientes). En individuos inmunosuprimidos puede producirse el síndrome de hiperinfección al producirse la migración de las larvas, con manifestaciones intestinales y pulmonares más graves, fiebre, debilidad y con mayor número de larvas en heces y también en esputo. Los factores de riesgo más importantes son, entre otros, los tratamientos inmunosupresores por anti-TNF, corticoesteroides, trasplantes de órganos sólidos o hematopoyéticos, cáncer e infección por HTLV-1 (Schär et al., 2013). Las larvas pueden atravesar la barrera hematoencefálica produciendo encefalitis y tasas de mortalidad de hasta el $87 \%$. Llegados a este punto, habitualmente el tratamiento utilizado para la estrongiloidiasis ya no es efectivo (Buonfrate et al., 2015), por ello, es esencial un cribado de individuos con sospecha de posible estrongiloidiasis antes de un tratamiento inmunosupresor (Schär et al., 2013).

El tratamiento de elección de la estrongiloidiasis se realiza con Ivermectina $200 \mu \mathrm{g} / \mathrm{kg} /$ día durante dos días; el alternativo con Albendazol 400 mg cada 12 horas durante dos días (Henriquez-Camacho et al., 2016).

El método de diagnóstico de referencia (gold standard) es la observación al microscopio de larvas rabditiformes en preparaciones de muestras fecales utilizando la técnica de Baerman (Buonfrate et al., 2015). La problemática de este 
CARMEN RODRÍGUEZ DIEGO; PEDRO FERNÁNDEZ-SOTO; ANTONIO MURO ÁLVAREZ APLICACIÓN DEL MÉTODO STRONG-LAMP PARA LA DETECCIÓN MOLECULAR DE STRONGYLOIDES STERCORALIS EN UNA POBLACIÓN DE PORTO IGUAZÚ, MISIONES, ARGENTINA

método es que presenta una muy baja sensibilidad ( $\leq 30 \%$ ) (Montes et al., 2010) y requiere en ocasiones de muestras seriadas (Buonfrate et al., 2015). Los métodos serológicos presentan problemas de especificidad. Los métodos moleculares están basados en la PCR, poco estandarizados, caros y técnicamente complejos de realizar. Una alternativa es la técnica de amplificación isotérmica de ácidos nucleicos tipo LAMP (Loop-mediated isothermal amplification) descrita por Notomi et al. (2000). El método LAMP se basa en el principio de síntesis de ADN por desplazamiento de cadena, llevado a cabo por una Bst polimerasa con elevada actividad de desplazamiento (Bacillus stearothermophylus) a temperatura entre 60-65 ${ }^{\circ} \mathrm{C}$, y un sistema de 4 o 6 cebadores: dos internos, Forward Inner Primer (FIP) y Backward Inner Primer (BIP); dos externos (F3 y B3), y, opcionalmente, 2 cebadores bucle o loop primers (FLP y FLB), diseñados para acelerar la reacción mediante la unión adicional a sitios no accesibles a los cebadores internos. En total, se reconocen 6 a 8 secuencias distintas en el ADN diana, confiriendo a la técnica una gran especificidad. Las ventajas fundamentales que presenta este método con respecto a otros métodos moleculares son que no requiere aparataje sofisticado, ya que puede realizarse en un simple termobloque, y que la visualización de los resultados se realiza por cambio de color o aparición de turbidez (Li et al., 2017). En el Centro de Investigación de Enfermedades Tropicales de la Universidad de Salamanca (CIETUS), se ha desarrollado previamente un método LAMP para la detección sensible y específica de ADN de Strongyloides spp. (Strong-LAMP). Se ha comprobado su funcionamiento en un modelo experimental de infección en el laboratorio y, hasta la fecha, también con éxito en un reducido número de muestras de heces de pacientes (Fernández-Soto et al., 2016).

\section{2. Овjetivos}

\subsection{Objetivo general}

Aplicación y valoración del método Strong-LAMP en muestras de heces de población de Porto Iguazú, Misiones, Argentina, conservadas en papel de filtro.

\subsection{Objetivos específicos}

1. Obtención de ADN de larvas 3 de $S$. venezuelensis.

2. Extracción de ADN de muestras de heces conservadas en papel de filtro.

3. Análisis de las muestras mediante Strong-LAMP. 
CARMEN RODRÍGUEZ DIEGO; PEDRO FERNÁNDEZ-SOTO; ANTONIO MURO ÁLVAREZ APLICACIÓN DEL MÉTODO STRONG-LAMP PARA LA DETECCIÓN MOLECULAR DE STRONGYLOIDES STERCORALIS EN UNA POBLACIÓN DE PORTO IGUAZÚ, MISIONES, ARGENTINA

\section{MATERIALES y MÉTODOS}

\subsection{Muestras de heces de pacientes}

Se han utilizado 100 muestras de heces recolectadas de población residente en barrios periurbanos y/o comunidades selváticas de desmonte de Puerto Iguazú en el Departamento de Iguazú (Provincia de Misiones) (Figura 1), dentro de un proyecto de investigación financiado por la Fundación Mundo Sano y realizado durante los años 2017-2019.

FIGURA 1. Mapa de Argentina mostrando la zona de estudio Ubicación geográfica de la provincia de Misiones y de la aldea Fortín Mbororé
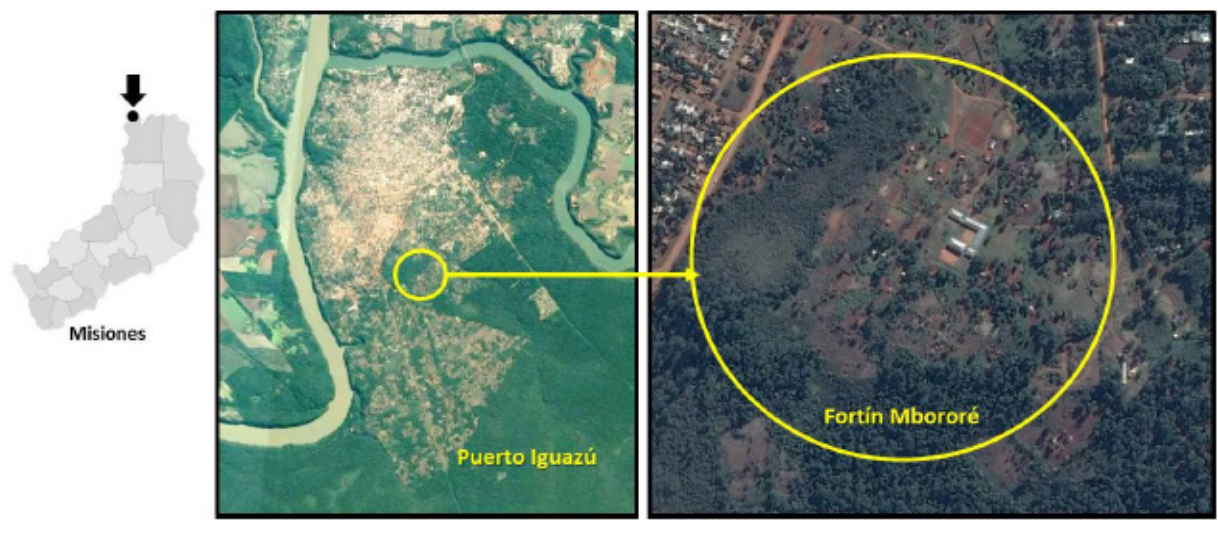

\subsection{Obtención del ADN de las muestras}

\subsubsection{Obtención y preparación de ADN de Strongyloides venezuelensis}

Como control positivo de amplificación en las reacciones de Strong-LAMP se utilizó $\mathrm{ADN}$ de larvas $\mathrm{L} 3$ de $S$. venezuelensis, cuyo ciclo biológico se mantiene en ratas Wistar infectadas experimentalmente en el CIETUS. Para la extracción del ADN a partir de las larvas se utilizó el kit comercial Nucleo Spin Tissue Kit (Machery-Nagel), siguiendo las instrucciones del fabricante. Posteriormente, se midió la concentración del ADN obtenido en un espectrofotómetro NanoDrop (ND-1000) y se ajustó a una concentración final de $5 \mathrm{ng} / \mu \mathrm{L}$. El ADN obtenido $(2 \mu \mathrm{L})$ se utilizó como control positivo de las distintas reacciones realizadas. 
CARMEN RODRÍGUEZ DIEGO; PEDRO FERNÁNDEZ-SOTO; ANTONIO MURO ÁLVAREZ APLICACIÓN DEL MÉTODO STRONG-LAMP PARA LA DETECCIÓN MOLECULAR DE STRONGYLOIDES STERCORALIS EN UNA POBLACIÓN DE PORTO IGUAZÚ, MISIONES, ARGENTINA

3.2.2. Obtención y preparación de $\mathrm{ADN}$ de heces conservadas en papel de filtro

Tras la recolección de las muestras de los pacientes, se tomó una porción de cada una y se extendió en un papel de filtro $(10$ x $2 \mathrm{~cm})$, se dejó secar al aire, se dobló a la mitad y se envolvió individualmente en papel de aluminio para su conservación a temperatura ambiente hasta que fueron remitidas al CIETUS.

La extracción y obtención de ADN se llevó a cabo siguiendo la metodología descrita por Cevallos et al. (2018) para muestras de heces conservadas en papel de filtro. Se utilizó un kit comercial: $i$-genomic Stool DNA Extraction Mini Kit (Intron Biotechnology), siguiendo las instrucciones del fabricante. El ADN obtenido $(2 \mu \mathrm{L})$ se utilizó como molde en las reacciones de amplificación mediante Strong-LAMP.

\subsection{Análisis parasitológico de muestras de heces}

En el laboratorio de la Sede Operacional de Mundo Sano (Puerto Iguazú) se realizó un análisis microscópico de las 100 muestras para la posible detección de formas parasitarias, incluyendo protozoos y geohelmintos. Para la detección de S. stercoralis, además, se realizó la técnica de Baerman para la recuperación de larvas (Baermann, 1917).

\subsection{Análisis molecular de muestras de heces mediante Strong-LAMP}

Todas las muestras de heces incluidas en el estudio se analizaron mediante el método LAMP descrito y puesto a punto previamente en el CIETUS: el StrongLAMP (Fernández-Soto et al., 2016). La mezcla de reacción utilizada se indica en la Tabla 1. Todas las reacciones se incubaron durante $60 \mathrm{~min}$ a $63^{\circ} \mathrm{C}$ en un termobloque $\mathrm{K}$ Dry-Bath ${ }^{\circledR}$, más 10 min a $80^{\circ} \mathrm{C}$ para desactivar la enzima y parar la reacción. Se utilizaron como molde de amplificación $2 \mu \mathrm{L}$ de $\mathrm{ADN}$ de cada muestra de heces. Como control positivo de amplificación se utilizó en todas las reacciones ADN de $S$. venezuelensis $(2 \mu \mathrm{L})$ y como control negativo agua $(2 \mu \mathrm{L})$ en lugar de ADN.

\subsection{Detección de los productos de amplificación}

El revelado de las muestras se realizó postamplificación, añadiendo a las reacciones $2 \mu \mathrm{L}$ de una dilución 1:10 de colorante SYBR Green I (10,000X; Invitrogen). Las muestras que resultaron positivas viraron su color a verde y las negativas mantuvieron el color naranja original del colorante.

Ediciones Universidad de Salamanca / @@ఠ $\quad$ FarmaJournal, vol. 5, núm. 2 (2020), pp. 73-82 
CARMEN RODRÍGUEZ DIEGO; PEDRO FERNÁNDEZ-SOTO; ANTONIO MURO ÁLVAREZ APLICACIÓN DEL MÉTODO STRONG-LAMP PARA LA DETECCIÓN MOLECULAR DE STRONGYLOIDES STERCORALIS EN UNA POBLACIÓN DE PORTO IGUAZÚ, MISIONES, ARGENTINA

Tabla 1. Mezcla de reacción utilizada en el Strong-LAMP La enzima Bst polimerasa, buffer y $\mathrm{MgSO} 4$ fueron suministrados por New England Biolabs; la betaína por SIGMA y los dNTPs por Intron

\begin{tabular}{|c|c|}
\hline Componentes & Volumen $(\mu \mathrm{L})$ \\
\hline $\mathrm{H}_{2} \mathrm{O}$ & 12.7 \\
\hline dNTPs $(10 \mathrm{mM})$ & 3.5 \\
\hline Isothermal Buffer $10 \mathrm{x} \mathrm{MgSO}_{4}(100 \mathrm{mM})$ & 2.5 \\
\hline $\mathrm{F} 3(10 \mathrm{pmol} / \mu \mathrm{L})$ & 1.5 \\
\hline $\mathrm{B} 3(10 \mathrm{pmol} / \mu \mathrm{L})$ & 0.5 \\
\hline $\mathrm{FIP}(100 \mathrm{pmol} / \mu \mathrm{L})$ & 0.5 \\
\hline $\mathrm{BIP}(100 \mathrm{pmol} / \mu \mathrm{L})$ & 0.4 \\
\hline Bst polimerasa $2.0 \mathrm{WS}$ & 0.4 \\
\hline DNA $(0.5 \mathrm{ng} / \mu \mathrm{L}) \mathrm{o} \mathrm{H}_{2} \mathrm{O}$ & 1 \\
\hline Total & 2 \\
\hline
\end{tabular}

\section{Resultados}

\subsection{Análisis parasitológico}

El análisis coproparasitológico de las muestras permitió la observación directa de larvas de S. stercoralis en 7/100 (7 \%) muestras analizadas (n. ${ }^{\text {os }: ~ 25, ~ 57, ~ 105, ~}$ $106,108,110$ y 133$)$.

\subsection{Análisis molecular mediante Strong-LAMP}

Los resultados obtenidos tras analizar las muestras de heces de los pacientes mediante Strong-LAMP se muestran en la Figura 2. Se obtuvo resultado positivo de amplificación (color verde) en 39/100 (39\%) muestras analizadas (n. ${ }^{\circ}: 33,72$, $74,109,129,105,106,110,155,136,141,142,143,161,6,10,34,118,170,173$, $57,54,133,134,4,18,31,80,15,132,5,12,44,37,83,64,103,73,104)$. De las muestras que resultaron positivas mediante Strong-LAMP, solamente 5 (n. ${ }^{\text {os: }}$ 105, 106, 110, 57 y 133) habían resultado también positivas parasitológicamente. Sin embargo, 2 muestras resultaron negativas mediante Strong-LAMP aunque habían resultado parasitológicamente positivas $\left(n .{ }^{\mathrm{os}}: 108\right.$ y 25$)$. 
FiguRa 2. Análisis mediante Strong-LAMP de las muestras de heces

$\mathrm{S}, \mathrm{ADN}$ de $S$. venezuelensis (control positivo); N, control negativo (agua

ultrapura). Color verde indica un resultado positivo; el naranja, negativo. Los círculos rojos indican aquellas muestras con resultado coproparasitológico positivo

\begin{tabular}{|c|c|c|c|c|c|c|c|c|c|c|c|}
\hline s & (57) & 54 & (133) & 134 & 4 & 18 & 31 & 36 & 80 & 82 & $\mathrm{~N}$ \\
\hline ? & a & 1. & & 18 & 4 & 18 & 1 & 4 & $y$ & $y$ & $y$ \\
\hline$S$ & 7 & 15 & 27 & 132 & 32 & 9 & 13 & 35 & 29 & 28 & $\mathrm{~N}$ \\
\hline$y$ & & & & & & & $y$ & $y$ & 1 & 1 & 4 \\
\hline 5 & 22 & 5 & 11 & 30 & (25) & 12 & 20 & 19 & 21 & 16 & $\mathrm{~N}$ \\
\hline f & $y$ & $y$ & I & & $y$ & 4 & $y$ & $y$ & d & al & 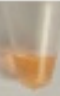 \\
\hline$s$ & 44 & 47 & 78 & 37 & 79 & 48 & 83 & 64 & 81 & 77 & $\mathrm{~N}$ \\
\hline$=$ & 3 & 3 & $=$ & $\exists$ & $=$ & $\theta$ & 6 & $E$ & 5 & $\Rightarrow$ & $E$ \\
\hline$S$ & 103 & 76 & 43 & 73 & 104 & 55 & 38 & 84 & 75 & 71 & $\mathrm{~N}$ \\
\hline 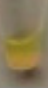 & $x$ & 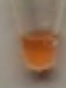 & $=$ & 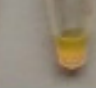 & $x$ & & 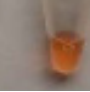 & 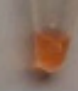 & & 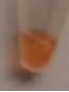 & 5 \\
\hline
\end{tabular}

\section{Discusión}

En la actualidad, la carencia de un verdadero diagnóstico de certeza sigue siendo la principal problemática de la estrongiloidiasis. El análisis coprológico, considerado como el gold standard, presenta una muy baja sensibilidad (Montes et al., 2010). Existen métodos diagnósticos alternativos, como los serológicos, pero presentan problemas de especificidad debidos a reactividad cruzada con otras infecciones por helmintos y su sensibilidad se puede ver disminuida en pacientes inmunocomprometidos y en áreas endémicas (Puthiyakunnon et al., 2014). Los métodos moleculares, como las PCR, son más sensibles, pero son caros y requieren personal cualificado (Buonfrate et al., 2015). La falta de un diagnóstico estandarizado genera una subestimación de la prevalencia de la enfermedad.

En este trabajo analizamos mediante el método molecular Strong-LAMP, desarrollado para la detección de ADN de S. stercoralis (Fernández-Soto et al., 2016), un total de 100 muestras. Hasta 39 muestras (39\%) han resultado positivas mediante Strong-LAMP, incluyendo 5 muestras positivas por análisis 
coproparasitológico. Así, el método Strong-LAMP ha resultado ser más sensible que el análisis parasitológico para la detección de S. stercoralis. Sin embargo, dos de las 100 muestras resultaron positivas parasitológicamente, pero negativas al análisis por Strong-LAMP. La presencia de falsos negativos puede ser debida a la baja carga parasitaria en infecciones crónicas, que no se encuentre material nucleico en la porción de muestra extendida en el papel de filtro o a la presencia de inhibidores (proteasas bacterianas, nucleasas, restos celulares y ácidos biliares) (Buonfrate et al., 2015). Además, este método ya ha resultado ser útil para la detección de $S$. stercoralis en muestras de heces humanas (Fernández-Soto et al., 2016) y en la detección de otros parásitos como Amphimerus sp. en zona endémica utilizando papel de filtro como método de conservación de las muestras de heces (Cevallos et al., 2017). Los datos de prevalencia obtenidos con el método Strong-LAMP (39 $\%)$, en comparación con los coproparasitológicos (7 \%), apuntan a que el método de diagnóstico molecular Strong-LAMP podría ser utillizado como una herramienta potencialmente útil en el diagnóstico de la estrongiloidiasis y podría usarse en combinación con métodos parasitológicos en áreas endémicas para establecer con mayor fiabilidad la prevalencia real de la enfermedad.

\section{Conclusiones}

1. ${ }^{a}$ El método molecular Strong-LAMP ha resultado más sensible que los métodos de diagnóstico parasitológicos para la detección de ADN de Strongyloides stercolaris en muestras de heces de pacientes.

2. ${ }^{a}$ El papel de filtro es un soporte válido para conservar y almacenar las muestras de heces a temperatura ambiente para la posterior extracción de $\mathrm{ADN}$ de Strongyloides stercoralis.

\section{Bibliografía}

Baermann GT. Eine einfache methode zur auffinfung von ankylostomun (nematoden) larven in erdproben. Laboratories Weltevreden Feestbundel, Mededeel mith H. Geneesk. Batavia; 1917. pp. 41-47.

Buonfrate D, Formenti F, Perandin F, Bisoffi Z. Novel approaches to the diagnosis of Strongyloides stercoralis infection. Clin Microbiol Infect. 2015; 21(6):543-552.

Cevallos W, Fernández-Soto P, Calvopiña M, Buendía-Sánchez M, López-Abán J, Vicente B. et al. Diagnosis of amphimeriasis by LAMPhimerus assay in human stool samples long-term storage onto filter paper. PLoS One. 2018; 13(2):e0192637.

Cevallos W, Fernández-Soto P, Calvopiña C, Fontecha-Cuenca C, Sugiyama H, Sato M. et al. LAMPhimerus: A novel LAMP assay for detecting Amphimerus sp. DNA in human stool samples. PLos Negl Trop Dis. 2017; 11(6):e0005672. 
CARMEN RODRÍGUEZ DIEGO; PEDRO FERNÁNDEZ-SOTO; ANTONIO MURO ÁLVAREZ

APLICACIÓN DEL MÉTODO STRONG-LAMP PARA LA DETECCIÓN MOLECULAR DE STRONGYLOIDES STERCORALIS EN UNA POBLACIÓN DE PORTO IGUAZÚ, MISIONES, ARGENTINA

Faust EC, Russell PF, Jung RC. Nematodos fasmidios parásitos del hombre. En: Craig y Faust, editores. Parasitología clínica; 1983. pp. 284-296.

Fernández-Soto P, Sánchez-Hernández A, Gandasegui J, Bajo Santos C, Lopez-Abán J, Saugar JM et al. Strong-LAMP: A LAMP Assay for Strongyloides spp. Detection in Stool and Urine Samples. Towards the Diagnosis of Human Strongyloidiasis Starting from a Rodent Model. Plos Neglect Trop D. 2016; 10(7):e0004836.

Henríquez-Camacho C, Gotuzzo E, Echevarría J, White AC Jr, Terashima A, Samalvides $\mathrm{F}$ et al. Ivermectin versus albendazole or thiabendazole for Strogyloides stercoralis infection. Cochrane Database Syst Rev. 2016; (1):CD007745.

King SE, Mascie-Taylor CG. Strongyloides fuelleborni kellyi and other intestinal helminths in children from Papua New Guinea: associations with nutritional status and socioeconomic factors. PnG. Med. J. 2004; 47:181.

Li Y, Fan P, Zhou S, Zhang L. Loop-mediated isothermal amplification (LAMP): A novel rapid detection platform for pathogens. Microb Pathog. 2017; 107:54-61.

Montes M, Sawhney C, Barros N. Strongyloides stercoralis: there but not seen. Curr Opin Infect Dis. 2010; 23(5):500.

Notomi T, Okayama H, Masubuchi H, Yonekawa T, Watanabe K, Amino N et al. Loop-mediated isothermal amplification of DNA. Nucleic Acids Res. 2000; 28(12):E63.

Puthiyakunnon S, Boddu S, Li Y, Zhou X, Wang C, Li J, Chen X. Strongyloidiasis-An Insight into Its Global Prevalence and Management. PLoS Negl Trop Dis. 2014; 8(8):e3018.

Schär F, Trostdort U, Giardina F, Khieu V, Muth S, Vounatsou P et al. Strongyloides stercoralis: Global Distribution and Risk Factors. Plos Neglect Trop D. 2013; 7(7):e2288.

Ediciones Universidad de Salamanca / @@ఠ $\quad$ FarmaJournal, vol. 5, núm. 2 (2020), pp. 73-82 